
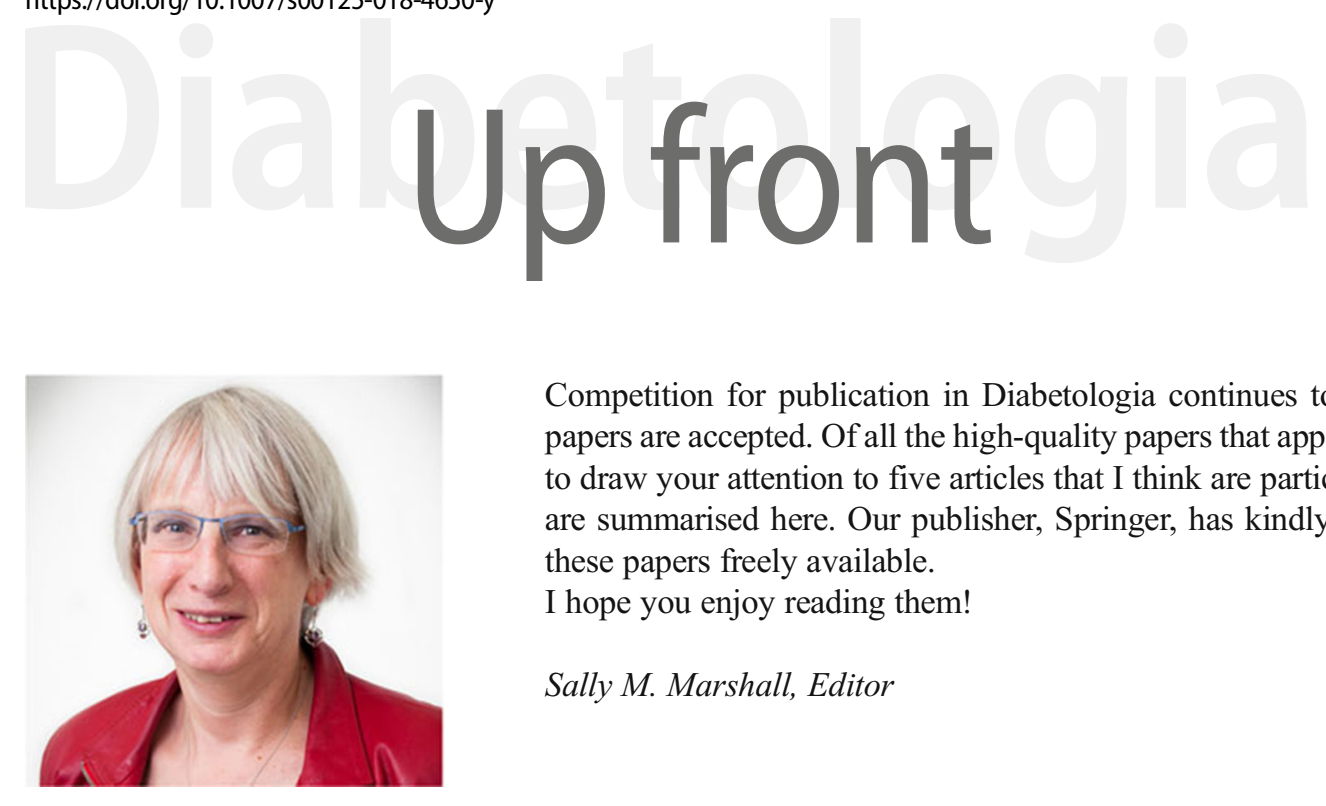

Competition for publication in Diabetologia continues to grow, and less than $20 \%$ of papers are accepted. Of all the high-quality papers that appear in this month's issue I want to draw your attention to five articles that I think are particularly interesting. The articles are summarised here. Our publisher, Springer, has kindly made the full text of each of these papers freely available.

I hope you enjoy reading them!

Sally M. Marshall, Editor

\section{Endocrine-disrupting chemicals and risk of diabetes: an evidence-based review}

P. Monica Lind and Lars Lind

Over recent decades, a large number of environmental chemicals that are present at detectable levels in the majority of people who live in the industrialised world have been reported to potentially act as endocrine-disrupting chemicals (EDCs). EDCs can interfere with any aspect of hormone action, including regulation of hormone synthesis, secretion or receptor binding. In this issue, Lind and Lind (https://doi.org/10.1007/ s00125-018-4621-3) review the current evidence suggesting that EDCs may be involved in the development of diabetes. The authors report moderate-strength evidence that exposure to dichlorodiphenyldichloroethylene ( $p, p^{\prime}$-DDE), a persistent metabolite of the pesticide dichlorodiphenyltrichloroethane (DDT), is involved in diabetes development. However, the strength of the evidence was poorer for other groups of EDCs, such as dioxins, polychlorinated biphenyls (PCBs), perfluorinated substances (PFASs), brominated flame retardants and bisphenol A, either because of a lack of large prospective studies or a lack of supportive experimental data. The authors suggest that the association between EDCs and diabetes may be strong enough to be considered as a serious problem by the European Chemicals Agency, REACH. However, other than for $p, p^{\prime}$-DDE, further evidence is required to support the association between EDCs and diabetes.

(b) The figure from this review is available as a downloadable slide.
Diabetes in the older patient: heterogeneity requires individualisation of therapeutic strategies

Guntram Schernthaner, Marie Helene Schernthaner-Reiter

Due to the improved life expectancy, about one third of all individuals with diabetes worldwide are older than 65 years. In a review in this issue, Schernthaner and SchernthanerReiter (https://doi.org/10.1007/s00125-018-4547-9) describe the enormous heterogeneity of the older population with diabetes, who range from otherwise healthy individuals to frail individuals with comorbidities, including cardiovascular disease (CVD), heart failure and/or renal impairment. The authors warn of the risks of very strict glucose control, which potentially causes more harm than benefit, particularly in patients with a limited life expectancy. The advantages of modern glucose-lowering drugs that do not induce (severe) hypoglycaemia are discussed in detail. For patients with established CVD, the authors state that the documented benefit of some drugs in cardiovascular outcome trials (CVOTs) should be considered in the treatment decision. About half of the patients in these trials were older than 65 years, and the documented benefit was more pronounced for some drugs (empagliflozin, canagliflozin, exenatide) in these older patients. The authors conclude that a carefully designed individual approach is needed for the treatment of older patients with type 2 diabetes, in which health status, presence or absence of complications, and life expectancy should be taken into account. (b) The figure from this review is available as a downloadable slide. 
Community-based pre-pregnancy care programme improves pregnancy preparation in women with pregestational diabetes

Jennifer M. Yamamoto, Deborah J. F. Hughes, Mark L. Evans, Vithian Karunakaran, John D. A. Clark, Nicholas J. Morrish, Gerry A. Rayman, Peter H. Winocour, Clare Hambling, Amanda W. Harries, Michael J. Sampson, Helen R. Murphy

Pre-pregnancy care is associated with improved pregnancy outcomes in women with diabetes. However, most women with diabetes do not attend specialist pre-pregnancy services. In this issue, Yamamoto et al (https://doi.org/10.1007/s00125018-4613-3) report the development and evaluation of a community-based pre-pregnancy care programme. The East Anglia programme was designed to target all women with diabetes aged 16-45 years, regardless of whether or not they intended to start a family, and to increase awareness among primary care providers. After implementation, women with type 2 diabetes had improved measures of pregnancy preparation: they were more likely to take preconception folic acid and to reach target $\mathrm{HbA}_{1 \mathrm{c}}$ levels. Measures of pregnancy preparation were above national averages both for women with type 1 or type 2 diabetes. The authors conclude that prepregnancy care advice should be community-based and targeted at all women of reproductive years.

Global burden of hypoglycaemia-related mortality in 109 countries, from 2000 to 2014: an analysis of death certificates

Francesco Zaccardi, Nafeesa N. Dhalwani, David R. Webb, Melanie J. Davies, Kamlesh Khunti

The negative impact of hypoglycaemia on glucose control and quality of life in individuals with diabetes is well known. In the last decade, however, observational studies and post hoc analyses of randomised controlled trials have also suggested a possible link between severe hypoglycaemia and risk of death. In this issue, Zaccardi et al (https://doi.org/10.1007/s00125018-4626-y) describe the global burden of hypoglycaemiarelated mortality in more than 100 countries between 2000 and 2014. Using information on the underlying cause of death reported in death certificates, the authors report global differences among countries and over time. The authors propose that such geographical and temporal patterns might be related to heterogeneous causes, including differences in socioeconomic development or inappropriate intensive glucose treatment in older individuals with diabetes, and suggest alternative strategies to reduce the global burden of hypoglycaemiarelated mortality.

Patterns of differential gene expression in a cellular model of human islet development, and relationship to type 2 diabetes predisposition

Marta Perez-Alcantara, Christian Honoré, Agata WesolowskaAndersen, Anna L. Gloyn, Mark I. McCarthy, Mattias Hansson, Nicola L. Beer and Martijn van de Bunt

Genome-wide association studies have identified $>100$ loci associated with type 2 diabetes risk. Whilst evidence points to a key role for pancreatic islets, the influence of islet development in type 2 diabetes remains largely unexplored. In this issue, Perez-Alcantara, Honoré et al (https://doi.org/10.1007/s00125-018-4612-4) show how gene expression in an islet differentiation model points to potential novel regulators of beta cell development, and highlight how these may influence type 2 diabetes predisposition. They report that type 2 diabetes loci, as a whole, are enriched for genes most highly expressed in the most mature stage of pancreas development, whilst other loci peak during an intermediate stage of development. These findings suggest that genetic risk factors for type 2 diabetes affect both mature islets and developing beta cells.

All text supplied by the authors. 